\title{
Das Messiasgeheimnis und die Spruchquelle
}

\author{
Walter Schmithals \\ Von Humboldt Universität Berlin \\ Deutschland
}

\begin{abstract}
The Messianic Secret and the Sayings Source $Q$

The article represents a new way of looking at the complexity with regard the "Messianic Secret" as a tribute to William Wrede, who was born almost 150 years ago. Wrede advocated a literary and historical solution to the Messianic Secret found in the Synoptic Gospels. The article aims to resolve the riddle by also taking the Sayings Source $Q$ into consideration. $Q$ is seen as located in the disciple group of John the Baptist and this group's adherents. The article argues that Mark developed the Messianic Secret as theme to adapt the unmessianic message of $Q$. As effect, Mark initiated a "christological" use of this motif within the church. The thesis of the article represents a modification that is a progression of some of the author's earlier opinions.
\end{abstract}

\section{EINLEITUNG}

Am 23 November 2006 jährte sich zum 100sten Mal der Todestag von William Wrede, ohne dass, wenn ich recht sehe, davon Notiz genommen wurde. Das mag damit zusammenhängen, dass die evangelisch-theologische Fakultät in Breslau, an der Wrede gelehrt hatte, seit 1945 nicht mehr existiert.

Bedauerlich ist es allemal; denn Wrede hat ein Erbe hinterlassen, das sich die Wissenschaft vom Neuen Testament noch keineswegs hinreichend angeeignet hat. W Bousset urteilte 1907 nach dem frühen Tod seines Freundes:

Er hatte einen Widerwillen dagegen, sich in ausgefahrenen
Geleisen zu bewegen, er war immer misstrauisch gegen
vermeintlich allgemein anerkannte Wahrheiten und Dogmen, die
sich, ohne dass sie begründet waren, in seine Wissenschaft
einzuschleichen drohten. Er hielt sich nicht der Mühe für
überhoben, da von neuem die Fundamente zu überprüfen, wo die
meisten fröhlich weiterbauten; er hatte den Mut von vorne
anzufangen .... Nur wenige stimmten dem einsam, abseits von den
Schlagworten der Parteien Wandelnden unbedingt zu, sehr viele 


\section{Das Messiasgeheimnis und die Spruchquelle}

ärgerten sich an inm, aber alle fühlten, dass man an diesen Leistungen nicht vorübergehen durfte.

(Bousset 1907:4-5)

Sich an Wrede zu erinnern täte einer Zeit gut, in der die Wissenschaft vom Neuen Testament sich weithin in ausgefahrenen Geleisen bewegt und mancherlei Konsens pflegt, der auf keinem soliden Fundament steht. Vielleicht ist 150 Jahre nach seinem Geburtstag, nämlich am 10 Mai 2009, die Zeit gekommen, sich des unerledigten Erbes von William Wrede intensiver zuzuwenden.

Der vorliegende Beitrag wendet sich dem bedeutsamsten Thema zu, mit dem „von vorne anzufangen“ Wrede 1901 den Mut hatte, nämlich dem Rätsel, vor das „Das Messiasgeheimnis in den Evangelien“ (Wrede 1901) den Erforscher des Neuen Testaments nach wie vor stellt. Wredes Auflösung dieses Rätsels hat sich zwar nicht als haltbar erwiesen; sie ist aber bis heute nicht durch eine überzeugendere und allgemein anerkannte Lösung ersetzt worden. Der vorliegende Aufsatz ruft meinen eigenen Versuch zur Lösung des Rätsels (Schmithals 1979) in Erinnerung, indem er die Spruchquelle thematisch in die Untersuchung einbezieht und damit einen bisher meines Wissens unbegangenen Weg zur Lösung des Rätsels beschreitet.

\section{DAS MESSIASGEHEIMNIS}

Unter dem Begriff „Messiasgeheimnis“ fasste Wrede bekanntlich eine Reihe miteinander korrespondierender Motive zusammen, die sich besonders im ältesten Evangelium in markanter Weise erkennen lassen (vgl. im Einzelnen Schmithals 1985:422-427). Einerseits hält Jesus seine Messianität und die Notwendigkeit seines Todes und seiner Auferstehung bis zu seiner Ankunft in Jerusalem öffentlich geheim, wobei die Schweigegebote an die Dämonen, die als numinose Wesen über Jesu Messianität informiert sind, ein besonders auffälliges Charakteristikum dieses Motivs darstellen („Messiasgeheimnis“ im engeren Sinn). Zum anderen belehrt Jesus einerseits seine engsten Vertrauten Petrus, Jakobus und Johannes, andererseits den Kreis seiner zwölf Jünger vertraulich über seine Würde und das damit verbundene heilvolle Geschick von Kreuz und Auferstehung („esoterische Jüngerbelehrung"). Schließlich zeigen sich die Jünger diesen Belehrungen gegenüber wenig verständnisvoll („Jüngerunverständnis“), auch wenn Jesus innen den messianischen Sinn der Gleichnisreden, die vor der Öffentlichkeit verhüllende Reden sind, im esoterischen Kreis offenbart („Gleichnistheorie“).

Die zu Wredes Lebzeiten vorherrschende „liberale“ Leben-Jesu-

Theologie, die sich für ihre Biographie Jesu vor allem auf das Markusevangelium stützte, hatte diese Motive psychologisch im Zusammenhang mit einer Entwicklung der Persönlichkeit Jesu zu deuten 
versucht: Jesus sei in Aufnahme seiner prophetischen Berufung zunehmend zu der Überzeugung gelangt, dass er der Messias sei, und er habe seine Jünger in pädagogischer Weisheit allmählich mit seinem Persongeheimnis und seinem zu erwartenden Schicksal vertraut gemacht. Wrede kritisiert diesen Versuch, die genannten Motive für eine Rekonstruktion des Lebens Jesu fruchtbar zu machen, als unwissenschaftlich, weil man zuerst den literarischen Sinn des Motivkomplexes entschlüsseln müsse. Er hält die zusammenhängenden Motive für eine Anschauung der frühen Gemeinde, die einen Ausgleich zwischen ihrem Wissen um den unmessianischen Verlauf des Lebens Jesu und ihrem österlichen Messiasbekenntnis herstellen wollte. Den Schlüssel für diese Deutung findet Wrede in dem Schweigegebot Markus 9:9, wo Jesus nach seiner „Verklärung“ den Jüngern gebot, „, ,iemand zu erzählen, was sie gesehen hatten, bevor der Menschensohn von den Toten auferstanden sei“. Der Evangelist Markus habe den ganzen Motivkomplex, ohne inn noch zu verstehen oder zu deuten, einfach mit seinen Überlieferungen weitergegeben. Wredes scharfsinniges Buch beschäftigt bis heute die Ausleger des Neuen Testaments (vgl. z B Bousset 1902:307-316, 347-362; Blevins 1982).

Indessen erkannte die von $\mathrm{M}$ Dibelius und $\mathrm{R}$ Bultmann initiierte formgeschichtlich orientierte Forschung bald, dass der Motivkomplex des Messiasgeheimnisses nicht in den älteren Überlieferungen, sondern erst in den Rahmenstücken des Markusevangeliums zu finden ist, so dass man ein Interesse des Evangelisten selbst an diesen Motiven in Rechnung zu stellen hat. Die redaktionsgeschichtlich orientierte Forschung bestätigte diese Erkenntnis, und das Interesse, das der Evangelist mit seiner Theorie verband, fand man nun in der Regel im Rahmen einer anspruchsvollen theologischen Deutung, die sich an Erkenntnissen der „dialektischen“ Theologie orientierte, wie sie sich nach dem Ersten Weltkrieg durchsetzte. Danach wollte der Evangelist Markus mit seiner Geheimnistheorie den paradoxen Charakter der Christusoffenbarung dartun: Das Geheimnis dieser Offenbarung enthüllt sich nur für den Glauben; dem Unglauben bleibt es verschlossen, so dass man trotz sehender Augen nicht sieht und versteht. Das Messiasgeheimnis ist letzten Endes also das Ostergeheimnis, das jeweils neu dem Glauben die Bedeutung Jesu als des gekreuzigten Christus enthüllt (vgl. z B Ebeling 1939; Kollmann 1991:267-273; Schnelle 2005:253-257).

Man berief sich für diese christologische Metatheorie gerne wie in anderer Weise schon Wrede auf Jesu Schweigegebot in Markus 9:9, das bis Ostern in Geltung stehen sollte, übersah dabei aber wie auch Wrede, dass Jesus selbst sich schon vor dem Hohen Rat demonstrativ zu seiner Messianität bekennt (Mk 14:61-64) und dass dies öffentliche Bekenntnis von großem Gewicht ist, den weiteren Verlauf der Passionsgeschichte bestimmt 
und zu Jesu Verurteilung führt. ${ }^{1}$ Dieser Widerspruch, den Wrede hinnehmen konnte, weil er den Evangelisten nur mit unverstandenen Bruchstücken der Überlieferung umgehen sah, widerlegt die auf das Ostergeschehen focussierte theologische Deutung des Motivkomplexes als einer originalen Leistung des Evangelisten selbst. Man hat auch nicht bedacht, dass das Messiasgeheimnis auf Palästina beschränkt ist. Weilt Jesus im Heidenland, wo auch Petrus zum ersten Mal das Christusbekenntnis ausspricht (Mk 8:29), wird es nicht beachtet (Mk 7:24-30; 31-37), während Jesus es in Jerusalem sogleich selbst aufhebt und damit seinen Tod provoziert (Mk 14:61-64; vgl. schon Mk 10:6). Markus 14:61-64, nicht Markus 9:9 ist der wahre Schlüsseltext der Messiasgeheimnistheorie. ${ }^{2}$ Offensichtlich geht Markus davon aus, dass das Geheimnis der Person Jesus so lange gewahrt bleiben musste, wie Jesus öffentlich im jüdischen Land wirken wollte; denn auf die Aufhebung des Geheimnisses folgte zwingend das Todesurteil. Wir haben es also mit einer planvollen und, wie Wrede insofern richtig gesehen hat, mit einer historisierenden, nicht einer theologisierenden Konstruktion des Evangelisten zu tun. Jedenfalls ist es unwissenschaftlich, angesichts ihrer Rätselhaftigkeit das Vorhandensein der markinischen Geheimnistheorie überhaupt zu leugnen (z B Pesch 1976:48-63, 135-136; Pesch 1977:36-47), beziehungsweise zu ignorieren (Marxsen 1956) oder diese zu bagatellisieren und in unzusammenhängende Einzelmotive aufzulösen (z B Hengel 1983:221-265, 237-241; Lührmann 1987). Und man sollte sie auch nicht „auf den Kopf stellen“, wie Klumbies $(2001: 310)^{3}$ dies versucht. $^{4}$

\footnotetext{
${ }^{1}$ Das Schweigegebot in Mk 9:9 ist zwar Bestandteil der markinischen Geheimnistheorie, die in deren Rahmen singuläre Terminierung auf das Ostergeschehen verdankt sich indessen der Tatsache, dass es sich bei der Verklärungsgeschichte um eine ursprüngliche Ostererzählung handelt, deren Versetzung in die vorösterliche Situation der Evangelist mit seiner Terminangabe kaschiert (vgl. schon Julius Wellhausen 1909:71).

2 An Mk 14:61-64 scheitert auch die apologetische Deutung der Geheimnistheorie, die zuerst Martin Dibelius (1933:231-232) vorgetragen hat: Markus wolle erklären, wie Jesus trotz seiner messianischen Wirksamkeit habe gekreuzigt werden können; die Kreuzigung sei nur möglich gewesen, weil er seine Würde geheim gehalten habe. Aber gerade die Offenbarung seiner Würde führt zu seiner Verurteilung!

${ }^{3}$ „Es geht nicht um die Verschleierung eines mit siener Person verknüpften Geheimnisses.“ Vielmehr versuche Jesus zu verhindern, dass die Menschen inn seiner normalen Menschlichkeit berauben und „aus der Gemeinschaft seiner Mitmenschen“ herausheben (267), weil er sonst „an dem Mechanismus von Erhöhung und Erniedrigung" (266) zugrunde gehen musste. Am ende stirbt der markinische Jesus an dem Vernichtungspotential, das dem Wechselspiel von Erhöhung und Erniedrigung innewohnt, einem Mechanismus, den zu überwinden er gerade aufgetreten war. Der „Automatismus von Erhöhung und anschließender Erniedrigung" (267) durch die Menschen scheint mir freilich eine Erfindung von Klumbies zu sein. Und wieso Jesus unter diesen Umständen der Frage des Hohenpriesters, ob er der Christus sei, zustimmen und sich damit selbst ins Unrecht setzen konnte, statt sie zu verneinen, bleibt völlig rätselhaft.

${ }^{4}$ Unhaltbar dünkt mich auch die originelle Deutung von Gerd Theißen (1995:225-245), die freilich die Gleichnistheorie weitgehend unbeachtet lassen muss und natürlich auch die Spruchquelle nicht einbezieht: Die Geheimnistheorie soll den Christen ein gutes Gewissen machen, wenn sie ihren Glauben vor der Öffentlichkeit verbergen, und zugleich Mut zum Bekenntnis machen, sobald ihre christliche Identität bekannt wird. Aber die Ansicht, dass die Gemeinde, die direkt zum offenen Bekenntnis aufgefordert (Mk 4:21-22; vgl 3:13-15; 4:14; 6:7-13), indirekt bzw. verhüllt zugleich mit einem guten Gewissen versehen wird, wenn sie ihr Bekenntnis verbirgt, ist schwerlich ein geglückter Versuch, die Geheimnistheorie zu erklären.
} 
Der historisierende Anlass der Geheimnistheorie enthüllt sich auf dem von Wrede vorgezeichneten Weg definitiv, allerdings als eine Theorie des Evangelisten selbst, wenn man die Spruchquelle mit in die Betrachtung einbezieht. Dies hat Wrede unterlassen, und darin sind inm, habe ich nichts übersehen, alle seine Kritiker bis in unsere Tage gefolgt.

\section{GEHEIME EPIPHANIE IN DER SPRUCHQUELLE}

Die in Lukas 10:21-24 und Matthäus 11:23-27; 13:16-17 aus der Spruchquelle $Q$ übernommene Logienkombination enthält die markinische Geheimnistheorie.

Beide Evangelisten überliefern an dieser Stelle den Wortlaut der Spruchquelle in weitgehender Übereinstimmung, während nur Lukas die ursprüngliche Kombination als solche und deren Stellung im Anschluss an die Aussendungsrede in Lukas 10:2-16 bewahrt hat. In der Spruchquelle dürfte etwa der folgende Text zu lesen gewesen sein:

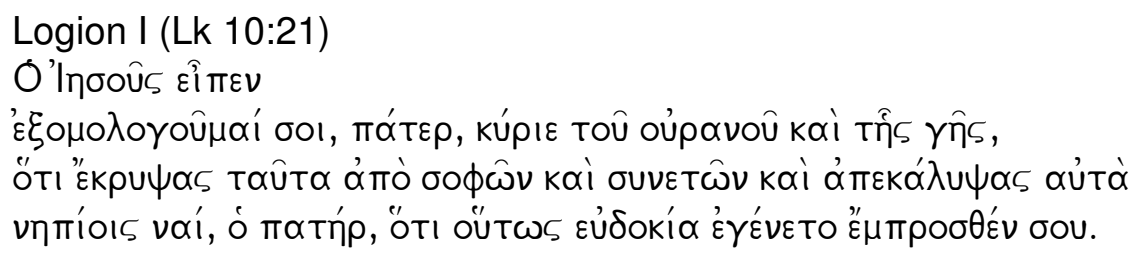

Bei Logion I und III handelt es sich um Prophetensprüche, die als solche aus der älteren Spruchüberlieferung in die Spruchquelle Q übernommen worden sind.

Logion I enthält einen Lobpreis. Gott wird einerseits als gütiger Vater, andererseits als machtvoller Herr angeredet. Der Gedanke des Spruchs ist weit verbreitet; „denn Gott widersteht den Hochmütigen, aber den Demütigen gibt er Gnade" (Spr 3:34; vgl. z B 1 Petr 5:5; Ps 8:3; Ps 116:6; Amos 7:14; Sap 10:21; Sir 3:19-30; Lk 1:52-53; Mk 10:13-15; Mt 18:4; 1 Kor 1:26-31). In 


\section{Das Messiasgeheimnis und die Spruchquelle}

der Spruchüberlieferung sind die „Unmündigen“ die Frommen, die dem Bußruf Jesu angesichts der anbrechenden Gottesherrschaft folgen, von der soeben die Rede gewesen war (Lk 10:9.11). Die „Klugen und Weisen“ dagegen sind die anerkannten Theologen, die schriftgelehrten Lehrer Israels, die „dieses Geschlecht" repräsentieren, das Johannes den Täufer und Jesus gleichermaßen verwirft (Lk 7:33-35), und denen die Weherufe gelten (Lk 11:754).

Logion III umfasst Lk 10:23b-24 und ist formal eine Seligpreisung. Selig gepriesen werden die Nachfolger Jesu, die seine Botschaft von der kommenden Gottesherrschaft annehmen und darum bei der bevorstehenden Äonenwende sehen werden, was die frommen Propheten und Könige Israels nur angesagt und erwartet haben. Auch dieses Motiv ist traditionell und entspricht zum Beispiel Ps Sal 17:44: „Selig sind, die in jenen Tagen leben, weil sie das Gut Israels sehen ..." (vgl. Hebr 11:13; 1 Petr 1:10). Schon jetzt sind den Nachfolgern Jesu die dämonischen Mächte untertan, und Jesus selbst sah den Satan vom Himmel stürzen wie einen Blitz (Lk 10:17-19; vgl. Lk 10:9; Lk 11:20).

Das Logion II fällt nicht nur aus dem Rahmen dieser beiden Prophetensprüche, sondern aus der Logienüberlieferung der Spruchquelle $Q$ überhaupt völlig heraus. Wir haben es mit einem hellenistischen Offenbarungswort zu tun, das vielfältige Parallelen in christlicher und in außerchristlicher Überlieferung besitzt (vgl. z B Joh 1:18; 3:35; 10:14-15; 17:25; Gal 4:4-5). Es benutzt die bekannte Vorstellung, dass Gleiches nur von Gleichem erkannt wird (vgl. 1 Kor 2:11). Der unbekannte Gott sendet einen inm wesensgleichen Offenbarer, den „Sohn“, dem er „alles“ übergeben und damit alle Vollmacht gegeben hat und durch den er deshalb sich, sein Wesen oder seinen Willen, vollkommen bekannt macht. Auffällig und ungewöhnlich ist in der vorliegenden Fassung dieses verbreiteten Gedankens, dass nicht nur von der Erkenntnis und Offenbarung des Vaters durch den Sohn, sondern auch und sogar in erster Linie von der Erkenntnis beziehungsweise der Offenbarung des Sohnes selbst die Rede ist. Den Sohn kennt als solchen beziehungswiese als den Offenbarer nur der Vater sowie derjenige, dem der

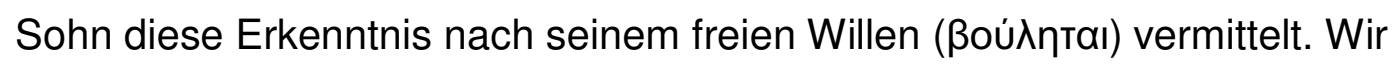
stoßen also im Rahmen eines im Übrigen traditionellen Offenbarungswortes auf das aus dem Markusevangelium bekannte Messiasgeheimnis.

Damit rücken aber auch die beiden anderen Logien in das Licht dieser christologischen Aussage. Logion I ist durch die erste Zeile von Logion II, die von der 1. Person in die 3. Person überleitet, eng mit diesem verbunden, so dass es sich im vorliegenden Zusammenhang bei dem taûta, das nur den Unmündigen offenbart ist, um das Persongeheimnis Jesu handeln muss. Dementsprechend erfahren und erkennen die in Logion III angesprochenen 
Jünger, dass der Messias, auf den viele Propheten und Könige warteten, in der Person Jesu erschienen ist. In der Einleitung von Logion III (Lk 10:23a), die dies Logion redaktionell an die vorangehende christologische Aussage anschließt, begegnet dabei expressis verbis das markinische Motiv der esoterischen Jüngerbelehrung. Das Geheimnis der Person Jesu offenbart

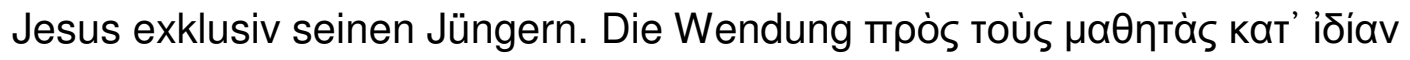
entspricht wortgleich den gleichsinnigen Aussagen, mit denen der Evangelist Markus die esoterische Belehrung der Jünger einführt (Mk 4:34; vgl. 6:31; 9:2, 28; 13:3). Auch das ungewöhnliche absolute ò matŕp und ò viòs von Logion II findet sich übrigens innerhalb der synoptischen Tradition nur noch in Markus 13:32, und zwar in einer analogen Aussage, so dass insofern gleichfalls die Hand desselben Schriftstellers am Werk gewesen sein dürfte.

Wie dem auch sei - der Redaktor der Logienkombination Lukas 10:2124 verwendet überlieferte Logien, um die Geheimnistheorie des Evangelisten Markus in der Spruchüberlieferung selbst zu verankern. Da nicht zu erkennen ist, warum eine spätere Hand zentrale Motive der markinischen Messiasgeheimnistheorie in die Spruchquelle eingeführt haben sollte, ist der Schluss unausweichlich, dass der Evangelist Markus auch der Herausgeber und Redaktor der Spruchquelle gewesen ist und ein Interesse daran hatte, einzelne ihrer überlieferten Sprüche so zu interpretieren, dass sie zum Ausweis der geheimen Epiphanie Jesu dienen.

\section{CHRISTOLOGISCHE PASSAGEN DER SPRUCHQUELLE}

Diese zunächst überraschende Folgerung lässt sich bis zu einem gewissen Grade überprüfen, wenn man die christologischen Aussagen der Spruchquelle daraufhin untersucht, ob in innen das Messiasgeheimnis gewahrt bleibt, und zugleich fragt, ob sich in ihnen Bezüge zum Markusevangelium nachweisen lassen. Wir prüfen unter diesen beiden Gesichtspunkten die entsprechenden christologischen Aussagen in der Reihenfolge, in der wir sie bei Lukas begegnen und in der sie vermutlich in der Spruchquelle zu lesen waren.

\subsection{Die Predigt Johannes des Täufers (Lk 3:2b-4, 7-9, 16-17 und Mt 3:1-4, 7-10, 11-12)}

In der Ankündigung des kommenden Stärkeren durch Johannes den Täufer, der mit dem heiligen Geist taufen wird, bleibt dieser Stärkere, nämlich Jesus, anonym. Sie durchbricht also das Messiasgeheimnis Jesu nicht.

Bemerkenswert ist indessen, dass Vers 16 den Bericht des

Markusevangeliums voraussetzt. Denn wie die Parallele bei Matthäus zeigt, war in der Spruchquelle zu lesen, „er wird euch mit dem heiligen Geist und mit 


\section{Das Messiasgeheimnis und die Spruchquelle}

Feuer taufen“. Das nur in der Spruchquelle begegnende „und mit Feuer taufen“, mit dem zu dem folgenden Gerichtswort aus der Spruchüberlieferung übergeleitet wird, ist als Bild für das Gericht singulär und kann nur als Analogiebildung zu „mit den heiligen Geist taufen“ verstanden werden, das sich in Markus 1:8 findet. Mit Vers 16 begegnet in der Spruchquelle also die christologische Aussage aus Markus 1:7-8, durch die dem prophetischen Bußprediger Johannes die Rolle eines Vorläufers Jesu zugewiesen worden ist.

\subsection{Die Taufe Jesu (Lk 3:21-22 und Mt 3:13, 16-17)}

Da die folgende Versuchungsgeschichte der Spruchquelle voraussetzt, dass der Leser um die Würde Jesu als „Sohn Gottes“ weiß, muss auch in der Spruchquelle von der Berufung Jesu berichtet worden sein, die Markus 1:9-11 zufolge während seiner Taufe durch Johannes erfolgte. Zwar finden sich in dieser Perikope bei Matthäus und Lukas nur zwei wenig signifikante Übereinstimmungen gegenüber dem Markustext, die auf die Fassung dieser Perikope in der Spruchquelle zurückgehen könnten, ${ }^{5}$ aber da die Spruchquelle keinerlei andere entsprechende Überlieferung verrät, dürfte sie wie zuvor schon mit der Ansage des Geisttäufers Markus 1:7-8 auch der darauf folgenden Erzählung Markus 1:9-11 gefolgt sein. Und weil Markus anders als die sekundären Seitenreferenten Matthäus und Lukas keine

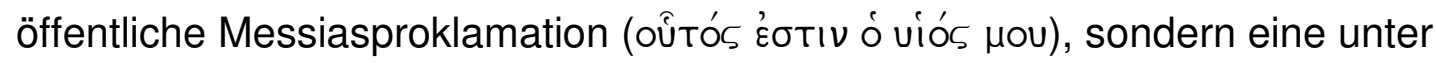
Ausschluss der Öffentlichkeit erfolgende Adoption Jesu zum „Sohn“ berichtet ( бù عî ó viós Hou), wird insofern wie bei Markus auch in der Spruchquelle das Messiasgeheimnis gewahrt worden sein.

\subsection{Die Versuchungsgeschichte (Lk 4:1-13 und Mt 4:1-11)}

In der markinischen Erzählung von dem 40 Tage lang währenden Aufenthalt Jesu in der Wüste (Mk 1:12-13) ist die Versuchung Jesu durch den Satan nur ein Nebenzug (Schmithals 1979:89-95). Dieser Nebenzug liegt indessen der ausführlichen Versuchungsgeschichte der Spruchquelle zugrunde, die demzufolge das Markusevangelium voraussetzt (Wellhausen 1909:74). Die Erzählung greift das markinische Motiv auf, wonach die dämonische Welt um die messianische Würde Jesu weiß (Mk 1:23-24; 5:6-7). Zwar handelt es sich nur bei der zweiten Versuchung um eine spezifisch messianische Versuchung, indem der Satan Jesus anbietet, inm die Weltherrschaft zu übertragen. Die Ansprache „Wenn du Gottes Sohn bist“ in Vers 3 und Vers 9 lässt aber keinen Zweifel daran, dass den Erzähler bei allen drei

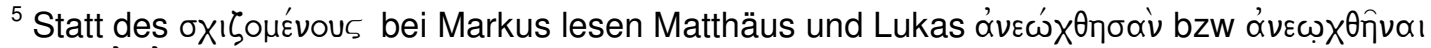

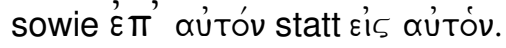


Versuchungen ein christologisches Interesse leitet. Der Teufel weiß als Angehöriger der numinosen Welt darum, dass Jesus bei seiner Taufe zum „Sohn Gottes" adoptiert wurde, und dem Leser wird auf die vorliegende Weise diese Hoheit aus berufenem Munde bestätigt. Er erfährt, dass in allem Folgenden nicht nur ein Prophet redet und handelt, sondern der Sohn Gottes. Dabei wird das Messiasgeheimnis gewahrt; denn natürlich findet keine der Versuchungen vor einem öffentlichen Forum statt.

\subsection{Der Hauptmann von Kapernaum (Lk 7:1-10 und Mt 8:5-13)}

Bei der Erzählung vom Hauptmann zu Kapernaum handelt es sich um die einzige ausgeführte Wundererzählung der Spruchquelle. Lukas hat sie stärker als Matthäus bearbeitet, doch interessieren die entsprechenden Differenzen im vorliegenden Zusammenhang nicht. Die Wundergeschichten des Markusevangeliums sind zwar Hinweise auf Jesu Messianität, wie der Evangelist in Markus 6:52 und 8:14-21 zu erkennen gibt, aber sie weisen als solche Jesus noch nicht als den Messias aus. Insofern durchbricht auch die vorliegende Erzählung das Messiasgeheimnis nicht, auch wenn in diesem Fall nicht, wie sonst in der Spruchquelle, der Lehrer und Prophet, sondern der „Herr" handelt.

Die Erzählung selbst ist eine Dublette zu Markus 7:24-30, der Perikope von der Syrophönizierin. Beide Male bittet ein Heide, beziehungsweise eine heidnische Frau, Jesus um Hilfe; beide Male geht es um einen Angehörigen des Bittenden; beide Male wehrt Jesus zunächst den Bittenden ab; beide Male wird eine Fernheilung berichtet; beide Male steht nicht Jesus als Wundertäter, sondern der bittende Heide im Mittelpunkt der Erzählung; beide Male rühmt Jesus den Glauben des Bittenden; beide Male fehlt der bei Wundergeschichten obligatorische Chorschluss . Offensichtlich ist die Erzählung vom Hauptmann von Kapernaum derjenigen von der Syrophönizierin nachgebildet. ${ }^{6}$ Sie wurde im Hinblick auf Jesu deutendes Wort erzählt: „... solchen Glauben habe ich selbst in Israel nicht gefunden“, auf das wir noch zurückkommen müssen.

\subsection{Die Anfrage des Täufers (Lk 7:18-23 und Mt 11:2-6)}

Diese Erzählung ist formal ein „Apophthegma“, also eine Szene mit einem abschließenden Wort Jesu, eine literarische Form, die der Spruchquelle im Übrigen ebenso wie die Wundergeschichten fremd ist. Inhaltlich geht sie auf die Aussage des Täufers zurück, dass nach inm ein Stärkerer kommt, der mit dem heiligen Geist und mit Feuer taufen wird (Lk 3:15-16 par). Ihr Thema ist also die Christologie, die erneut im Zusammenhang mit Johannes dem Täufer

\footnotetext{
${ }^{6}$ Vgl. auch die wörtlichen Berührungen in Mk 7:30 und Lk 7:10.
} 


\section{Das Messiasgeheimnis und die Spruchquelle}

in den Blick tritt. Das Messiasgeheimnis bleibt indessen gewahrt. Wenn selbst Johannes nicht weiß, dass Jesus der Messias ist, weiß auch kein anderer Mensch, was nur in der jenseitigen Welt bekannt ist. Und Jesus gibt auch eine bloß indirekte Antwort auf die Anfrage des Täufers, indem er auf seine Wundertaten verweist, die zwar ein Hinweis auf seine Hoheit, aber kein Beweis für seine Messianität sind. Ausdrücklich preist er deshalb denjenigen selig, der an inm keinen Anstoß nimmt, obschon er doch den messianischen Erwartungen vieler Frommer nicht entspricht, die den Messias als machtvolle Herrschergestalt erwarten. Damit wird letztlich offensichtlich auf Jesu Tod am Kreuz angespielt, der innerhalb der alten Spruchüberlieferung noch nicht als messianisches Leiden beziehungsweise als heilvolles Sterben begegnet, sondern als Prophetenschicksal (Lk 11:49-51par; 13:33-34par).

Der Verweis auf die Fülle seiner Wundertaten erscheint insofern in der Spruchquelle fehl am Platz, als die Spruchüberliefung Jesus zwar als Lehrer und Prophet, nicht aber als Wundertäter kennt. Lukas hat diese Unstimmigkeit empfunden und in den Bericht der Spruchquelle die Bemerkung eingefügt, Jesus habe, als die Boten des Johannes zu ihm kamen, gerade viele von Krankheiten und Leiden und bösen Geistern geheilt und vielen Blinden das Augenlicht geschenkt. Der Autor der Spruchquelle teilte solche Bedenklichkeit indessen nicht. Er blickte auf die Wundergeschichten des Markusevangeliums und setzte solche Kenntnis auch bei seinen Lesern voraus.

\subsection{Jesu Zeugnis über Johannes den Täufer (Lk 7:24-28 und Mt 11:7- 11)}

Hatte Johannes in Lukas 3:15-17par selbst von sich bezeugt, dass er nur Vorläufer des Stärkeren sei, der mit dem heiligen Geist und mit Feuer taufen wird, so legt nun Jesus ein entsprechendes Zeugnis über Johannes ab. Johannes ist mehr als ein Prophet, nämlich der im Alten Testament angekündigte letzte Bote Gottes vor dem Anbruch der Gottesherrschaft. Wer der Kommende ist, dem Johannes den Weg bereitet, bleibt ungesagt. Vers 27, auf den die entsprechende Aussage Jesu zuläuft, ist ein Mischzitat, das auf Mal 3:1 und 2 Mose 23:20 beruht. Sagt Gott aber in Mal 3:1, dass der Engel den Weg „vor mir“ bahnen soll, heißt es im vorliegenden Text „,vor dir“. Das Zitat wird damit zwar auf den Messias bezogen. Um wen es sich dabei handelt, erfährt der Hörer aber nicht. Das Messiasgeheimnis, das für den Leser natürlich kein Geheimnis mehr ist, bleibt also in der vorausgesetzten historischen Situation gewahrt. 
Das Mischzitat findet sich wörtlich gleichlautend ${ }^{7}$ auch in Markus 1:2, hier gleichfalls im Rahmen der Geheimnistheorie des Evangelisten und merkwürdigerweise Jesaja zugeschrieben. Matthäus und Lukas lassen das Zitat an dieser Stelle aus und bringen es später an der vorliegenden Stelle nur nach der Spruchquelle, womit auch jener Irrtum stillschweigend korrigiert und eine Dublette vermieden wird. Das ganz ungewöhnliche und so spezifisch modifizierte Zitat muss auf denselben Autor zurückgehen, dem es im Rahmen seiner Geheimnistheorie an beiden Stellen im Hinblick auf die Vorläuferrolle des Johannes (siehe unten) gleich wichtig war; es bezeugt also dieselbe Hand im Markusevangelium und in der Spruchquelle.

\subsection{Vom Ernst der Entscheidung (Lk 13:24-30 und Mt 7:13-14,22-23;} 8:11-12)

Diese kleine Rede Jesu stellt unterschiedliche Überlieferungen zusammen, die zum Teil der alten Spruchüberlieferung angehören dürften. Bei Lukas 13:25b-26 handelt es sich aber zweifellos um eine christologische Aussage, die Jesus mit dem Herrn des Gleichnisses, dem Weltenrichter, identifiziert: „.. wird er antworten und zu euch sagen: Ich kenne euch nicht, woher ihr seid. Dann werdet ihr sagen: Wir haben vor dir gegessen und getrunken und in unseren Gassen hast du gelehrt." Diese Passage ist sekundär in das Gleichnis vom Hausherrn eingelegt und sprengt dies Gleichnis als solches, wenn es auch das Geheimnis wahrt. Denn hier wird die bildhafte Rede verlassen, und es sprechen Menschen, die Jesus persönlich kennen gelernt haben, als er unter innen lebte und lehrte. Sie berufen sich auf inn, werden von ihm aber nicht anerkannt. Um wen es sich bei innen konkret handelt, bleibt ungesagt. Dass es die Juden überhaupt sind, ist schon angesichts von Lukas 13:28 unwahrscheinlich.

In Lukas 13:29-30 findet sich in diesem Zusammenhang wie schon in der Erzählung vom Hauptmann zu Kapernaum ein deutlicher Hinweis auf Menschen aus der heidnischen Welt, die mit den Erzvätern und den Propheten in die Gottesherrschaft eingehen, so dass Erste, die Jesus 13:25b26 zufolge in Palästina begegnet sind, zu Letzten und Letzte zu Ersten werden. Dieser abschließende Spruch von der Umwälzung aller Verhältnisse findet sich auch in Markus 10:31 und könnte von dort in den vorliegenden Zusammenhang übertragen worden sein.

\footnotetext{
${ }^{7}$ Mir der unbedeutenden Variante eines in der Spruchquelle (Lk 7:27b par) aus der

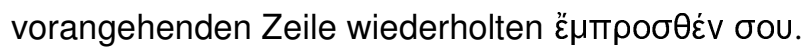




\section{Das Messiasgeheimnis und die Spruchquelle}

\subsection{Zusatz zur Wehklage über Jerusalem (Lk 13:35b und Mt 23:39)}

In der Spruchquelle hatte der Weheruf gegen die Prophetenmörder (Lk 11:4748par) einen doppelten Zusatz, den Lukas in dessen zweiten Teil, der Wehklage über Jerusalem, an einen anderen Ort versetzt und verselbständigt hat (Lk 11:49-51; 13:34-35 und Mt 23:34-39). Während der Weheruf die Nachkommen der Prophetenmörder in 2. Person anredet, spricht in dem Zusatz zunächst „die Weisheit Gottes“ in der 3. Person über diese Mörder, deren Schuld das gegenwärtige - letzte - Geschlecht des alten Äons zu büßen haben wird. Die folgende Klage über Jerusalem wählt dann wieder die Anredeform und blickt offenbar auf den sich vollziehenden Untergang Jerusalems im Jahre 70.

Das abschließende Wort der Weisheitsrede greift auf Markus 11:9, die Erzählung von Jesu Einzug in Jerusalem, zurück: „Ich sage euch aber: Ihr werdet mich nicht sehen, bis der Tag da ist, an dem ihr ruft: Gelobt sei, der da kommt im Namen des Herrn." Dieser für den Leser unmissverständliche Rückgriff auf einen Bericht des Markusevangeliums dient dazu, „die Weisheit Gottes“, die zuvor gesprochen hat, mit Jesus zu identifizieren, der als Messias in Jerusalem einzieht, eine Identität, die freilich in der vorausgesetzten Situation erst in der Zukunft und in Jerusalem festgestellt werden kann und darum zur Zeit der vorliegenden weisheitlichen Rede Jesu das Messiasgeheimnis wahrt.

\subsection{Das große Mahl (Lk 14:15-24 und Mt 22:1-14)}

Bei dem Gleichnis vom großen Mahl handelt es sich neben Lukas 19:1127par um eine der beiden Parabeln, die in der Spruchquelle begegnen. Die vorliegende Parabel setzt das Christusbekenntnis voraus; denn das eschatologische „Jetzt“ der Stunde, zu der die zuvor Eingeladenen aufgefordert werden, sich bei der Mahlzeit einzufinden, ist ohne Zweifel das „Jetzt“ der Zeit post Christum crucifixum et resurrexum, in der das Evangelium verkündigt wird. Durch den Gleichnischarakter der Aussage wird ohne weiteres das Messiasgeheimnis gewahrt, auch wenn die markinische Gleichnistheorie, die indessen vorausgesetzt sein dürfte, in der Spruchquelle nicht ausdrücklich begegnet. Im Übrigen ist ein unmittelbarer Bezug zum Markusevangelium nicht zu erkennen.

Das Thema der Parabel, das weitere Beachtung verdient, ist - wie in der Wundererzählung vom Hauptmann zu Kapernaum - der Zutritt der Heiden, die in den Ausgestoßenen, Ausgegrenzten und Auswärtigen abgebildet sind, zur christlichen Heilsgemeinde. 


\subsection{Das anvertraute Geld (Lk 19:11-27 und Mt 25:14-30)}

Die Parabel von dem anvertrauten Geld bildete allem Anschein nach den Abschluss der Spruchquelle, und es ist anzunehmen, dass sie einen gewichtigen Schlusspunkt setzen sollte. Ihre christologische Ausrichtung liegt am Tage; denn bei dem verreisenden Herrn, der seinen Sklaven einen Geldbetrag hinterlässt, damit sie inn gewinnbringend verwenden, und der wiederkommt, um Rechenschaft von innen zu fordern, handelt es sich ohne Frage um den erhöhten Herrn Jesus Christus, der wiederkommen wird, zu richten die Lebenden und die Toten. Wiederum wahrt die Form des Gleichnisses ohne weiteres das Messiasgeheimnis.

Die Rekonstruktion ihrer ursprünglichen Fassung und die Deutung der an herausgehobenen Ort platzierten Parabel sind allerdings nicht leicht. Die Allegorisierung, die Lukas vornimmt, indem er unter Rückbezug auf einen Bericht des Josephus (Ant 17:11) die Gestalt des Königs Archelaos einführt, der sich in Rom das Herrschaftsgebiet seines Vaters Herodes hatte übertragen lassen, ist zweifellos sekundär. Aber auch die Differenzierung in der Höhe der übertragenen Gelder durch Matthäus, der auf diese Weise die Parabel offensichtlich auf die unterschiedlichen Charismen in der Gemeinde bezogen wissen will, ist nicht ursprünglich. Sie fehlt bei Lukas, und ohne die bei Matthäus vorliegende Differenzierung ist die Deutung der übertragenen Gelder auf die Geistesgaben in der Parabel nicht angedeutet.

Den Schlüssel zur Deutung der ursprünglichen Parabel liefert indessen der abschließende Satz in Lukas 19:26par, mit dem zugleich die Spruchquelle selbst geendet haben dürfte und der in Markus 4:25 eine genaue Parallele besitzt: 8 Jedem, der hat, dem wird gegeben, aber von dem, der nicht hat, wird auch noch genommen werden, was er hat."

Diesem Logion liegt eine geläufige Redewendung zugrunde („Die Reichen werden immer reicher"; "Wo Tauben sind, da fliegen Tauben zu"), die auch in der Antike vielfach bezeugt ist. Die Fortsetzung, dass dem, der nicht hat, auch noch genommen wird, was er hat, ist dagegen unserem Logion eigentümlich und dürfte deshalb in Markus 4:25 und Lukas 19:26 par ein besonderes Gewicht haben.

In Markus 4:25 begegnet dies Logion im Rahmen der markinischen Gleichnistheorie (vgl. Schmithals 1979:227-247). Diese besagt zunächst, dass es sich bei Gleichnissen um eine verhüllende Rede handelt, deren Sinn, „das

\footnotetext{
${ }^{8}$ Mk 4:25:

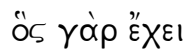

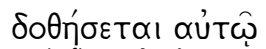

kai ös oủk हैXह1,

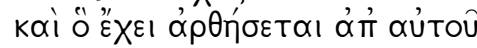

Lk 19:26:

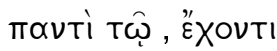

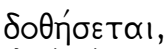

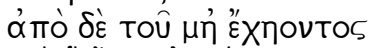

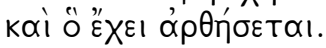




\section{Das Messiasgeheimnis und die Spruchquelle}

Geheimnis der Gottesherrschaft" (Vers 11), Jesus aber seinen Jüngern - nur innen - in esoterischer Belehrung enthüllt (Mk 4:10-12). Nachdem er dies im Blick auf das zuvor erzählte Gleichnis vom vierfachen Acker getan hat (Vers 13-20), erklärt Jesus in einem Bildwort (Vers 21) mit angeschlossener Deutung (Vers 22), ${ }^{9}$ dass die öffentliche Verhüllung der Gleichnisrede zeitlich limitiert ist; „denn nichts wird verborgen außer dazu, öffentlich zu werden“. Im Fortgang des Evangeliums wird der Leser sodann erfahren, dass Jesus selbst vor dem Hohen Rat das Geheimnis der Gottesherrschaft, nämlich seine Messianität, enthüllt und damit die zeitliche Begrenzung des Geheimnisses aufhebt (Mk 14:61-64).

Auf die Auskunft, das Geheimnis des Gottesreiches sei als solches zeitlich begrenzt, folgt in Markus 4 ein weiteres Bildwort (Vers 24b), ${ }^{10}$ das durch den Zusatz „und man wird euch noch mehr geben“ der folgenden Deutung gefügig gemacht wird. Diese Deutung (Vers 25) enthält unser Logion. Bildwort und Deutung werden der Aufmerksamkeit des Lesers besonders ans Herz gelegt: „Merkt auf das, was ich sage“ (Vers 24a). Im Kontext der markinischen Gleichnistheorie erschließt sich der Sinn des deutenden Logions leicht: Wer sich das „Geheimnis der Gottesherrschaft“, nämlich das Geheimnis der Person Jesu enthüllen lässt, kommt in den Besitz der vollen Wahrheit, andere dagegen, die es sich nicht enthüllen lassen, verlieren auch noch das, was sie von Jesus beziehungsweise der Gottesherrschaft zu wissen meinen.

Stellt man nun die Parabel von dem anvertrauten Geld, die mit unserem Logion endet, in das Licht dieser Sinngebung, muss es sich bei der Geldsumme, die ihr Herr den Sklaven, nämlich Christus seiner Gemeinde, zum „wuchern“ hinterlassen hat, um die Fülle des Evangeliums von dem Christus Jesus handeln, und das abschließende Wort der Verheißung und der Warnung sagt der diesem Evangelium gehorsamen Gemeinde die ganze Fülle des Heils zu, kündet anderen aber den Verlust auch noch dessen an, was sie zu besitzen meinen. Auf diesen Kreis von „anderen“ Hörern Jesu waren wir bereits in Lukas 13:25-27par gestoßen. Wer mit innen gemeint ist, wissen die Leser der Spruchquelle beziehungsweise des Markusevangeliums natürlich, während wir es aus dem Gesamtgefüge der Messiasgeheimnistheorie erschließen müssen (siehe unten).

Es ergibt sich, dass in der Spruchquelle überall dort, wo Jesus als der Christus begegnet, das Messiasgeheimnis gewahrt wird. Zugleich weisen die

\footnotetext{
${ }^{9}$ Beide Sprüche entnimmt er offenkundig der Logienüberlieferung, wie die Dubletten Lk 11:33 und 12:2 zeigen. ${ }^{10}$ Auch dieser Spruch stammt nach Ausweis der Dublette Lk 6:38 aus der
Logienüberlieferung.
} 
entsprechenden Aussagen in großer Regelmäßigkeit enge Bezüge zum Markusevangelium auf, und zwar Bezüge, die nicht auf die von der formgeschichtlichen Forschung vermuteten Einzelüberlieferungen zurückgehen, die sich wie fliegende Blätter hier und da niedergelassen haben sollen, sondern die literarischer Art sind. Wundergeschichte, Apophthegma und Parabel, wie sie als Träger christologischer Aussagen in der Spruchquelle begegnen, sind Formen, die dem Markusevangelium vertraut, der alten Spruchüberlieferung aber fremd sind. Damit bestätigt sich, dass der Evangelist Markus, der die verschiedenen Motive der Messiasgeheimnistheorie in seine Vorlagen eingebracht hat, auch der Herausgeber der Spruchquelle gewesen ist, deren ältere, noch unchristologische Überlieferungen offensichtlich erst von inm selbst durch die "geheimnisvollen“ christologischen Aussagen und Texte bereichert worden sind.

Dabei begegneten uns im Zusammenhang mit diesen christologischen Aussagen der Spruchquelle drei bemerkenswerte Motive, die bei der Lösung des Rätsels der Messiasgeheimnistheorie berücksichtigt werden müssen.

Einmal geht es um die Öffnung der Gottesherrschaft für die Heiden (Lk 7:1-10 par; 13:29 par; 14:15-24 par). Zum anderen weist der Autor mit Nachdruck Johannes dem Täufer die Rolle des Vorläufers Jesu zu (Lk 3:16 par; 7:18-23 par, 24-28 par). Schließlich treffen wir neben den Nachfolgern Jesu, denen das Geheimnis der Person Jesu voll erschlossen ist, auf andere, denen auch das noch genommen werden wird, was sie an Jesus zu haben meinen (Lk 13:24-30 par; 19:11-27 par).

\section{5. „DUBLETTEN“ UND „MENSCHENSOHNWORTE“}

Darüber hinaus sind noch zwei weitere Beobachtungen geeignet, bei der Lösung des Messiasgeheimnis-Rätsels behilflich zu sein.

„Dubletten“ nennen wir jene synoptischen Stoffe, die Matthäus und Lukas sowohl im Markusevangelium wie in der Spruchquelle gelesen haben. Sie sind schon oft und seit langem beobachtet, zusammengestellt und untersucht worden. Es handelt sich durchweg um Spruchüberlieferung, und bereits B Weiss (1897:468) urteilte mit guten Grund, dass „sich fast alle bei Markus außerhalb des Zusammenhanges seiner Erzählung aufbehaltenen Sprüche auf Reminiscenzen an Reden und Spruchreihen, deren Vorhandensein in der Quelle (sc Spruchquelle) zu konstatieren ist, zurückführen“ lassen. In Markus 12:37-40 (vgl. 4:2) gibt der Evangelist Markus zu erkennen, dass er das inm vorliegende Spruchgut nur auszugsweise verwendet; er "ist sich dessen bewusst, dass, was er hier bietet, nur Auswahl, nur Beispie/ ist“" (Dibelius 1933:237). In Markus 4:21-24 


\section{Das Messiasgeheimnis und die Spruchquelle}

verwendet Markus einige dieser Dubletten, wie wir gesehen haben, im Rahmen seiner Gleichnistheorie. Das in den „Dubletten“ begegnete Spruchgut ist durchweg unchristologisch und schon Wendling (1908: 36) hat beobachtet, dass die "Logienstoffe, die mit $Q$ verwandt sind, in Einschaltungen von der Hand des Redaktors auftauchen, während sie den älteren M(ar)k(us)-Stücken fremd sind".

Man kann also beobachten, dass derselbe Autor einerseits (unter Wahrung des Messiasgeheimnisses) christologische Stoffe und Aussagen in die Spruchquelle platziert, und dass er andererseits unchristologische Logien aus der Spruchüberlieferung in das Markusevangelium aufnimmt. Die beiden unterschiedlichen Traditionen werden also wechselseitig verschränkt.

Besonders aufschlussreich ist die Verwendung des Begriffs „Menschensohn" in der Spruchquelle und im Markusevangelium. Er entstammt der alten Logienüberlieferung, während er in den vorredaktionellen Stoffen des Markusevangeliums noch nicht begegnet. Bei dem

„Menschensohn" der alten Logienüberlieferung handelt es sich um den Weltenrichter, der überraschend und unberechenbar kommt, so dass allezeit Wachsamkeit vonnöten ist (Lk 12:40 par; vgl. 21:36), und der wie ein Blitz vom Himmel überall zugleich erscheinen wird, so dass niemand entfliehen kann (Lk 17:24 par). Seine Ankunft steht nahe bevor (Mt 10:23). Das Gericht, das er heraufführt, wird wie die Sintflut sein und wie Feuer und Schwefel, die Sodom vernichteten (Lk 17:26-27, 30 par). Die sich zu ihm bekennen, werden zwar in der Gegenwart verleumdet, verschmäht und aus der Synagoge ausgestoßen (Lk 6:22), aber zu innen wird sich auch der Menschensohn bekennen, wenn er zum Gericht kommt (Lk 12:8). Sie werden neben inm auf Thronen sitzen und die zwölf Stämme Israels richten (Mt 19:28 par). Wer die Propheten dazu auffordert, diese ihre Botschaft vom kommenden Menschensohn durch ein Zeichen zu legitimieren, wird schroff zurückgewiesen: Der Menschensohn selbst wird durch sein Kommen zum Gericht diese Botschaft legitimieren (Lk 11:30 par)! Wer etwas gegen inn sagt, kann allerdings Vergebung empfangen; ohne Vergebung aber bleibt, wer den prophetischen Geist lästert, der sein Kommen ansagt (Lk 12:10 par).

Der Evangelist Markus begegnet diesen von inm auszugsweise benutzten Aussagen der alten Spruchüberlieferung und versteht sie im Rahmen des Messiasgeheimnisses so (bzw will sie so verstanden wissen), dass Jesus verhüllend von seiner eigenen messianischen Hoheit spricht, wenn er das Kommen des "Menschensohns" ansagt. Nur wer in das Geheimnis der Person Jesu eingeweiht ist, versteht, dass Jesus sich selbst meint, wann immer er in dritter Person vom „Menschensohn“ wie von einem anderen redet. Nun ist der Begriff „Menschensohn“ bekanntlich doppeldeutig. 
Seine Verwendung für die messianische Richtergestalt bei der Äonenwende, wie sie in der Spruchüberlieferung zu beobachten ist, geht auf Daniel 7:13-14 zurück und findet sich in apokalyptischen Schriften des Judentums (äthHen 37-71; 4 Esdra 13). Im Übrigen bezeichnet „Sohn des Menschen“ einfach den einzelnen Menschen im Unterschied zum Kollektiv „Menschheit“. Diese Doppeldeutigkeit macht sich Markus zu Nutze, wenn er den Hoheitstitel "Menschensohn" in sein Evangelium einführt und sich dabei insofern streng an seine Vorlagen aus der Spruchüberlieferung hält, als er diese Bezeichnung ausschließlich im Munde Jesu begegnen lässt. Durch seine Doppeldeutigkeit wahrt der Begriff „Menschensohn“ das Messiasgeheimnis. Im Einzelnen begegnen wir bei inm Worten vom kommenden, denen vom leidenden und auferstehenden und denen vom irdisch wirkenden Menschensohn.

In Mk 8:38 und 13:26 redet Jesus in der Weise der Spruchüberlieferung in dritter Person vom kommenden Menschensohn, der auf den Wolken mit der Schar der Engel in der Herrlichkeit des Vaters erscheinen wird, eine Redeweise, die Markus beibehält, wenn Jesus sich in Mk 14:62 auf die Frage des Hohenpriesters, ob er der Christus sei, dazu bekennt, dass er selbst der Menschensohn seiner Verkündigung ist, den alle zur Rechten Gottes sitzen und mit den Wolken des Himmels kommen sehen werden. In diesem Schlüsseltext der Geheimnistheorie hebt Jesus demonstrativ das Geheimnis mit der Folge auf, dass der Hohe Rat inn wegen Gotteslästerung zum Tode verurteilt. Unter dem Kreuz bestätigt der heidnische Centurio, dass Jesus als der Christus/Menschensohn gestorben ist. $^{11}$

Zuvor hatte der Evangelist in auffälliger Manier Jesus im esoterischen Kreis der Jünger sein Leiden, Sterben und Auferstehen, also den Inhalt des zentralen christlichen Glaubensbekenntnisses, in der Weise voraussagen lassen, dass Jesus diese Ansagen in der verhüllenden dritten Person vom „Menschensohn“ macht (Mk 8:31; 9:9, 12, 31; 10:33-34, 45; 14:21, 41). Diese Ansagen versichern dem Leser, dass es sich bei dem in der Spruchüberlieferung begegnenden himmlischen Menschensohn-Richter und dem gekreuzigten und auferweckten Jesus Christus des kirchlichen Glaubensbekenntnisses um dieselbe Hoheitsgestalt handelt, und sie erklären zugleich, wieso diese Identiät während der galiläischen Wirksamkeit Jesu öffentlich verborgen bleiben konnte, zumal Jesu Worte vom Leiden und

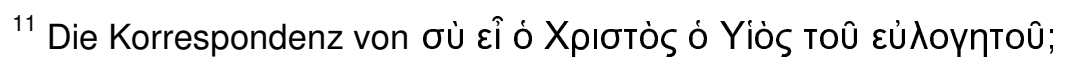

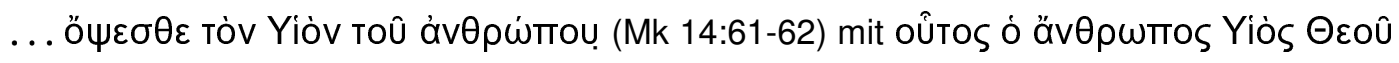
ก̂v (Mk 15:39) ist beziehungsvoll.
} 


\section{Das Messiasgeheimnis und die Spruchquelle}

Auferstehen „des Menschensohns“ sogar bei seinen engsten Jüngern auf Unverständnis stießen (Mk 9:32).

In Mk 2:10 und 2:28 ergänzt der Evangelist seine Vorlagen durch Aussagen vom „Menschensohn“, die sich auf Jesu irdisches Wirken beziehen und die Jesus vor aller Ohren machen kann, weil ihre Doppeldeutigkeit dennoch das Messiasgeheimnis wahrt. Denn wer mit der Feststellung gemeint ist, dass „der Menschensohn auf Erden Vollmacht hat, Sünden zu vergeben“ (Mk 2:10) und dass „der Menschensohn auch Herr ist über den Sabbat“ (Mk 2:28), bleibt im Zwielicht. Der Leser, der in das Geheimnis der Person Jesu eingeweiht ist, weiß, dass Jesus von sich selbst redet, der aktuelle Hörer aber hört Jesus von dem Menschen schlechthin sprechen. Der Titel „Menschensohn“ dient also einerseits dazu, das Messiasgeheimnis zu wahren, und zugleich sind diese beiden Stellen wegen ihrer Öffentlichkeit der deutlichste Hinweis auf die verhüllende beziehungsweise geheimnisvolle Art und Weise, in der Markus die Rede vom „Menschensohn“ als christologische Rede verstanden wissen will.

Neben diese beiden Stellen aus dem Markusevangelium sind in der Spruchquelle Lukas 7:34par und 9:58par zu stellen, wo Jesus sich gleichfalls im Hinblick auf sein irdischen Dasein und Wirken in aller Öffentlichkeit verhüllend als Menschensohn bezeichnet, der "isst und trinkt“ beziehungsweise „keinen Ort hat, wo er sein Haupt niederlegen kann“. Diese beiden Menschensohn-Aussagen sind in der Spruchquelle singulär, die im Übrigen nur die überlieferten Logien von dem mit Jesus nicht identischen, in der Zukunft kommenden Menschensohn-Richter kennt. Beide Stellen vom irdisch wirkenden Menschensohn müssen deshalb von der Hand des Evangelisten Markus stammen, der auf diese Weise sein Verständnis der Menschensohnworte auch in der Spruchquelle selbst verankert, so dass der Leser im Lichte von Lukas 7:34 par und 9:58 par erfährt, dass Jesus auch in den von der alten Spruchüberlieferung tradierten Worten vom kommenden Menschensohn von sich selbst gesprochen hat, wenngleich, seine Hoheit verhüllend, in dritter Person.

\section{DIE LÖSUNG DES RÄTSELS}

Alle bisherigen Beobachtungen verweisen auf eine Lösung des Rätsels, vor dem die Forschung angesichts der Messiasgeheimnistheorie des Evangelisten Markus steht, die auf dem von Wrede beschrittenen Weg zu suchen ist. Dem ältesten unserer Evangelisten geht es in der Tat, wie Wrede im Prinzip richtig gesehen hat, um einen Ausgleich zwischen messianischer und unmessianischer Jesusüberlieferung. Anders als Wrede vermutete, fand dieser Ausgleich jedoch nicht in einem frühen Stadium der urchristlichen 
Geschichte statt, so dass er zur Zeit des Evangelisten Markus bereits jede Aktualität verloren hatte. Vielmehr stand Markus selbst vor dem Problem, das Vorhandensein unmessianischer Jesusüberlieferung erklären zu müssen. Darum ist die Messiasgeheimnistheorie seine Theorie. ${ }^{12}$

Unmessianisch ist nämlich die in der Spruchquelle zusammengestellte alte Spruchüberlieferung, wie schon Harnack (1907:127, 162-163, 169) ${ }^{13}$ beobachtet hat. In diesen Überlieferungen redet Jesus als Prophet der nahen Endzeit (Lk 10:18), nicht als der Herr und Christus. Dementsprechend fehlen noch in der Spruchquelle die Passions- und Osterberichte, und es wurden auch keine Worte vom leidenden und auferstehenden Menschensohn gebildet. Zwar weiß diese Überlieferung offensichtlich um den Tod Jesu, deutet inn aber als Prophetengeschick (Lk 13:34-35 par). Jünger Jesu begegnen nicht, was dessen Wirksamkeit nicht als Rabbi, sondern als Prophet entspricht. Zu den Schriftgelehrten stehen die Tradenten vielmehr in schroffem Gegensatz (Lk 11:45-52 par). Johannes der Täufer und Jesus stehen zwar nebeneinander (Lk 7:31-34 par), aber da die Äonenwende mit Johannes dem Täufer angesetzt wird (Lk 16:16 par) und „unter denen, die von einer Frau geboren sind, niemand größer ist als Johannes“ (Lk 7:28 par), scheint Johannes der Täufer den höheren Rang einzunehmen.

Natürlich muss diese Gemeinde, die sich ausschließlich auf die apokalyptische Botschaft des Täuferkreises stützt, einen eigenen, von der österlichen Jüngergemeinde und dem folgenden Urchristentum unabhängigen Trägerkreis gehabt haben (vgl. z B Lührmann 1969:101; Kuhn 1970:308-309). Die lange Zeit herrschende Vorstellung, ein homogener Überlieferungsfonds sei in Spruchquelle und Markusevangelium zunächst separiert und später durch Matthäus und Lukas wieder zusammengefügt worden, ist historisch kaum vorstellbar und angesichts der unterschiedlichen theologischen Konzeptionen beider Überlieferungen unhaltbar. Wesentliche Stationen der Geschichte dieses Trägerkreises, einer „Täufergemeinde“ mehr als einer „Jesusgemeinde“, lassen sich aus der Spruchüberlieferung rekonstruieren (vgl. Schmithals 2004:429-454). Dass sie in Palästina zuhause war und dort die Botschaft des Täufers und die Predigt Jesu gehört hat, ergibt sich aus Lukas 13:26, wonach die Juden sich darauf berufen, dass Jesus in ihren Straßen gepredigt hat, sowie aus Matthäus 10:23, wo den Gemeindegliedern

\footnotetext{
${ }^{12}$ Angesichts der Tatsache, dass Markus unzweifelhaft einzelne Logien aus der unmessianischen Spruchüberlieferung in sein Evangelium aufgenommen hat, ist es unverständlich, dass Strecker (1979:196) so urteilen kann: „Da Markus mit Wahrscheinlichkeit unmessianische Jesusüberlieferung nicht gekannt hat, lässt sich die Geheimnistheorie im Rahmen seines Evangeliums auch nicht als Ausgleich von messianischer und unmessianischer Jesustradition verstehen."

${ }^{13}$ Beachtenswert ist in dieser Hinsicht aus der späteren Literatur vor allem die Untersuchung von Athanasius Polag (1977).
} 


\section{Das Messiasgeheimnis und die Spruchquelle}

geraten wird, bei Verfolgungen in eine andere Stadt zu fliehen, denn „ihr werdet mit den Städten Israels nicht fertig werden, bis der Menschensohn kommt“. Auch das Trostwort an die Verfolgten, die man „vor die Synedrien und in die Synagogen schleppt" und die dort zu ihrer Verteidigung auf die Eingebungen des Geistes vertrauen dürfen (Lk 12:11-12; Mk 13:11), verweist auf Palästina. Als Kaiser Caligula (37-41) im Jahre 40 plante, sein Standbild im Tempel zu Jerusalem aufstellen zu lassen, erwartete die Gemeinde in aktualisierter Naherwartung, dass durch diesen Frevel das Kommen des Menschensohnes und die Endkatastrophe ausgelöst und die Auserwählten erlöst werden würden (Mk 13:13b-20, 24-27) (Schmithals 1979:561-569, 583).

Die jüdische Katastrophe des Jahres 70 deuteten die prophetisch inspirierten Tradenten der Spruchüberlieferung in einem Wort, das von der "Weisheit Gottes" ausgesprochen wird, als Gericht über die Ermordung der Propheten durch die etablierten jüdischen Autoritäten, angefangen von Abel bis hin zu Sacharja, dem Sohn des Baruch, der dem Bericht des Josephus zufolge (Bell 4:334-344) zu Beginn des jüdischen Aufstandes mitten im Tempel von den Zeloten erschlagen worden ist (Lk 11:48-51 par). Wenn dieses Urteil von der "Weisheit“ ausgesprochen wird, zeigt sich indessen, dass der prophetische Enthusiasmus der Gemeinde zu dieser Zeit bereits erlahmt war. Ob sie die Katastrophe in Palästina selbst überlebt hat oder sich in Syrien hat in Sicherheit bringen können, wie es nach Angaben des Eusebius (KG III 5:3) die Jerusalemer Christengemeinde mit ihrer Flucht nach Pella getan haben soll, ist nicht zu erkennen.

Gegen die pharisäische Restauration der Synagoge nach der Zerstörung des Tempels setzen sich die Angehörigen der "Täufergemeinde“ verständlicherweise heftig zur Wehr, wie ihre Weherufe gegen die Pharisäer bezeugen (Lk 11:39-44 par). Folgerichtig werden sie aus der Synagoge ausgeschlossen, was sich aus dem Trostwort Lk 6:22-23 par ergibt, das die Frommen der Gemeinde selig preist, „wenn die Menschen euch hassen und euch ausschließen und euch verleumden wegen des Menschensohns".

Ob auch diejenige christliche Gemeinde, deren Traditionsgut, bereits als Evangelienschrift literarisch verfasst, der Evangelist Markus mit den Motiven seiner Messiasgeheimnistheorie verbindet und neu herausgibt, bis zur pharisäisch-rabbinischen Restauration dem Synagogenverband angehört hatte, wie man annehmen möchte, sei dahingestellt. Jedenfalls muss der Evangelist Markus in dieser Zeit in Verbindung mit der "Täufergemeinde“ gekommen sein. Während für die moderne Forschung die markinische Theorie des Messiasgeheimnisses das zu lösende Rätsel aufgibt, stand Markus vor dem Rätsel der unmessianischen Jesusüberlieferung. Die Geheimnistheorie ist seine Lösung dieses Rätsels.

Markus 14:61-64, der Schlüsseltext der Geheimnistheorie, besagt, dass nach Überzeugung des Evangelisten Markus Jesus seine messianische 
Hoheit verborgen halten musste, solange er wirken wollte; denn ein offenes Bekenntnis zu seiner Gottessohnschaft hätte unverzüglich zum Todesurteil wegen Gotteslästerung geführt. Da dieses Bekenntnis und die folgende Verurteilung erst in Jerusalem erfolgten, konnte der "Täufergemeinde“ in Galiläa, wo Jesus gewirkt hatte, Jesu messianische Hoheit verborgen bleiben. Damit vermochte sie auch nicht zu realisieren, dass der von Markus hoch verehrte Täufer Johannes (nur) der Vorläufer Jesu gewesen ist, ein Urteil, das, wie wir gesehen haben, Markus in der Spruchquelle intensiv zur Geltung bringt.

Natürlich geht es Markus nicht um die abstrakte Lösung eines vorgegebenen Problems. Er will offensichtlich die aus dem Synagogenverband ausgeschlossenen Angehörigen der "Täufergemeinde" für die eigene christliche Gemeinde gewinnen. Darum legt er das überlieferte Evangelium in der mit dem Messiasgeheimnis verknüpften Fassung den Lesern vor und akzeptiert zugleich in doppelter Weise die Logienüberlieferung der „Täufergemeinde“. Einerseits führt er diese in Gestalt der Spruchquelle in die kirchliche Jesusüberlieferung ein, indem er sie, gleichfalls mit dem Siegel des Geheimnisses versehen, messianisierte; andererseits nimmt er sie auszugsweise in sein Evangelium auf. Zugleich aber mahnt er die Angehörigen der „Täufergemeinde“, das Angebot der vervollständigten Wahrheit ihrer eigenen Überlieferung nicht zu verschmähen und nicht auf dem defizitären Verständnis der Logienüberlieferung zu beharren. Damit würden sie in Wahrheit auch das noch verlieren, was sie in der Nachfolge des Täufers und Jesu besitzen, und aus Ersten zu Letzten werden (Lk 13:24-30 par; 19:11-27 par).

Zugleich macht Markus, wie wir sahen, die Leser mit der Aufnahme von Heiden in die christliche Gemeinde vertraut. Dabei könnte schon eine Verbindung mit den heidenchristlichen Gemeinden paulinischer Observanz im Blick stehen. ${ }^{14}$ Es möchte aber auch an die gottesfürchtigen Heiden gedacht sein, die sich möglicherweise schon zuvor der judenchristlichen Gemeinde

\footnotetext{
${ }^{14}$ Manches spricht dafür, dass der Evangelist Markus selbst in diesem Heidenchristentum zuhause war (vgl Mk 2:19b; 8:38; 9:41, 50b; 10:12, 27, 45; 13:10; sowie Johannes Weiß [1903:94] und Martin Werner [1923]), während das seinem Evangelium zugrunde liegende Traditionsgut aus dem hellenistischen Judenchristentum stammt. Mit Vorlagen aus seinem eigenen Gemeindeverband hätte der Evangelist auch schwerlich so frei umgehen können, wie er es nach Ausweis der Geheimnistheorie tatsächlich tut. Und die historisch nicht haltbare Vorstellung der insoweit erst redaktionellen markinischen Passionsgeschichte, dass der Anspruch, der Messias zu sein, ein Todesurteil wegen Gotteslästerung zur Folge hat, ist einem Heidenchristen eher als einem Judenchristen zuzutrauen. Bemerkenswert ist auch, dass der Evangelist bereits von Verfolgungen durch römische Behörden ausgeht (Mk 13:9;

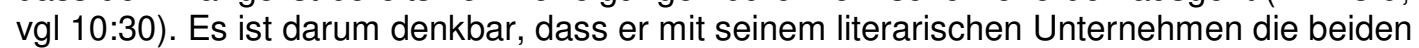
aus der Synagoge ausgeschlossenen Gruppen, die „Täufergemeinde“ und das hellenistische Judenchristentum, in der Gemeinschaft mit seiner heidenchristliche Gemeinde, die seit jeher außerhalb der Synagoge gelebt hat, zusammenführen wollte.
} 


\section{Das Messiasgeheimnis und die Spruchquelle}

angeschlossen hatten und die jedenfalls wie diese bei der pharisäischen Restauration die Synagoge verlassen mussten.

Es konnte Markus angesichts der unterschiedlichen Traditionsträger mit gutem Grund nicht einfallen, beide bis dahin getrennten literarischen Überlieferungen in einer einzigen Schrift zu vereinen. Spruchquelle und Markusevangelium finden indessen auf einer Buchrolle Platz, und es liegt nahe anzunehmen, dass beide Schriften von ihm in einem Konvolut veröffentlicht wurden. Daraus erklärt sich zwanglos, dass Matthäus ebenso wie Lukas unabhängig voneinander beide Schriften vorgefunden und das Bedürfnis empfunden haben, sie zu einem einzigen Werk zu vereinigen, was innen zugleich die redaktionelle Bearbeitung der Überlieferung erleichterte. ${ }^{15}$ Die Kopistenbemerkung in Markus 1:1 (vgl. z B Hos 1:1 LXX) dürfte in jenem Konvolut im Anschluss an die Spruchquelle den Beginn des Markusevangeliums als einer neuen Schrift markiert haben: „Anfang des Evangeliums von Jesus Christus“.

\section{Zitierte Literatur}

Blevins, J L 1982. The Messianic Secret in Markan Research, 1901-1976.

Washington, DC: University Press of America.

Bousset, W [1904] 1907. Vorwort zu W Wrede, Paulus. Halle/Tübingen.

Bousset, W 1902. Das Messiasgeheimnis in den Evangelien. ThR 5(8), 307-316, 347-362.

Dibelius, M [1933] 1971. Die Formgeschichte des Evangeliums. Tübingen: Mohr Siebeck.

Ebeling, H J 1939. Das Messiasgeheimnis und die Botschaft des MarcusEvangeliums. Berlin: Töpelmann.

Harnack, A 1907. Sprüche und Reden Jesu. Leipzig: J C Hinrichs.

Hengel, M 1983. In Stuhlmacher, P (Hrsg), Das Evangelium und die Evangelien. Tübingen: Mohr.

Klumbies, P-G 2001. Der Mythos bei Markus. Berlin: De Gruyter.

Kollmann, B 1991. Jesu Schweigegebote an die Dämonen. ZNW 82, 267-273.

Kuhn, H-W 1970. Der irdische Jesus bei Paulus als traditionsgeschichtliches und theologisches Problem. ZThK 67, 295-320.

Lührmann, D 1969. Die Redaktion der Logienquelle. Neukirchen-Vluyn: Neukirchener Verlag.

Lührmann, D 1987. Das Markusevangelium. Tübingen: Mohr Siebeck.

Marxsen, W 1956. Der Evangelist Markus. Zur Redaktionsgeschichte des Evangeliums. Göttingen: Vandenhoeck \& Ruprecht.

Pesch, R 1976. Das Markusevangelium, 1. Teil. Freiburg: Herder.

Pesch, R 1977. Das Markusevangelium, 2. Teil. Freiburg: Herder.

\footnotetext{
${ }^{15}$ Bekanntlich hat schon der Vater der Zwei-Quellen-Theorie, Christian Herman Weisse, mit gutem Grund vermutet, dass es sich bei den beiden Evangelienschriften, die Papias (Eusebius KG III 39) gekannt hat, gleichfalls um das Konvolut von Spruchquelle und Markusevangelium gehandelt hat.
} 
Polag, A 1977. Die Christologie der Logienquelle. Neukirchen: Neukirchener Verlag. Schmithals, W 1979. Das Evangelium nach Markus. 2 Bände. Gütersloh: Mohn.

Schmithals, W 1985. Einleitung in die drei ersten Evangelien. Berlin.

Schmithals, W 2004. Paulus, die Evangelien und das Urchristentum. Leiden: Brill.

Schnelle, U 2005. Einleitung in das Neue Testament. 5. Auflage. Göttingen: Vandenhoeck \& Ruprecht.

Strecker, G 1979. Zur Messiasgeheimnistheorie im Markusevangelium, in Pesch, R (Hrsg), Das Markusevangelium, 190-207. Freiburg: Herder. (WdF 159.)

Theißen, G 1995. Die pragmatische Bedeutung der Geheimnismotive im Markusevangelium. Ein wissenssoziologischer Versuch, in Kippenberg, H G \& Strousma, G G (eds), Secrecy and concealment: Studies in the history of Mediterranean and Near Eastern religions, 225-245. Leiden: Brill. (SHR 65.)

Weiss, B1897. Lehrbuch der Einleitung in das Neue Testament. Berlin.

Weiß, J 1903. Das älteste Evangelium. 3. Auflage. Göttingen: Vandenhoeck \& Ruprecht.

Wellhausen, J 1909. Das Evangelium Marci. 2. Auflage. Berlin: Reimer.

Wendling, E 1908. Die Entstehung des Marcus-Evangeliums. Tübingen: Mohr Siebeck.

Werner, M 1923. Der Einfluss der paulinischen Theologie im Markusevangelium. Giessen: Töpelmann.

Wrede, W [1901] 1913. Nachdrucke 1963, 1969. Das Messiasgeheimnis in den Evangelien: Zugleich ein Beitrag zum Verständnis des Markusevangelium. Göttingen: Vandenhoeck \& Ruprecht. [engl. Übersetzung durch J C G Greig, 1971, The Messianic Secret, Cambridge: J Clarke.] 